\title{
DESAFIOS PARA O DESENVOLVIMENTO DE PRODUTOS SUSTENTÁVEIS PARA A CONSTRUÇÃO
} CIVIL

Antônia Beatriz da Silva Teixeira (antonia.beatriz.silva06@aluno.ifce.edu.br) - Instituto Federal de Educação, Ciência e Tecnologia do Ceará - campus Quixadá, IFCE.

Mércia Maíra Araújo dos Santos (mercia.maira.araujo07@aluno.ifce.edu.br) - Instituto Federal de Educação, Ciência e Tecnologia do Ceará - campus Quixadá, IFCE.

Luiz Roberto Oliveira de Souza (luiz.roberto.oliveira06@aluno.ifce.edu.br) - Instituto Federal de Educação, Ciência e Tecnologia do Ceará - campus Quixadá, IFCE.

Joyce Custódio de Freitas (joyce.custodio@ifce.edu.br) - Instituto Federal de Educação, Ciência e Tecnologia do Ceará - campus Quixadá, IFCE.

Caio Augusto Nunes Marques (caio.marques@ifce.edu.br) - Instituto Federal de Educação, Ciência e Tecnologia do Ceará - campus Quixadá, IFCE.

\section{RESUMO}

A construção civil é uma indústria com elevado consumo de recursos naturais e com geração alta de resíduos. Com os impactos decorrentes da pandemia do novo coronavírus, evidenciou se ainda mais a crescente preocupação sobre a escassez dos recursos naturais, expondo as fragilidades dos modelos de desenvolvimento econômicos predominantes. A Economia Circular é apresentada como forma para reduzir esses impactos mantendo esses materiais em seu mais alto nível de utilidade e valor o tempo todo. Esse estudo teve como objetivo identificar os principais desafios para o desenvolvimento e utilização de produtos sustentáveis na construção civil. Para isso, foi desenvolvido um questionário online que foi aplicado junto a professores, engenheiros e arquitetos; em seguida identificou-se os termos mais recorrentes. Os principais empecilhos encontrados foram o preço dos produtos sustentáveis para os consumidores e a baixa consciência ambiental dos stakeholders. Existe a necessidade de uma maior cooperação entre setor privado, governos, comunidade científica e sociedade para que se estabeleça uma política ambiental eficaz que estimule o uso desses produtos. Os especialistas citam como alternativas a reeducação de todo o setor da construção civil, a ampliação dos estudos sobre essa temática e a elaboração de normas técnicas específicas para estes produtos.

Palavras chave: desenvolvimento Sustentável; economia circular; construção Civil 


\section{INTRODUÇÃO}

No ano de 2020 com a pandemia do novo coronavírus, o mundo sofreu consequências sociais e econômicas desastrosas, revelando fragilidades do sistema econômico mundial linear, consequentemente, proporcionando reflexões sobre tal sistema e a disponibilidade dos recursos do planeta (ELLEN MACARTHUR FOUNDATION, 2021).

De acordo com as estimativas da Global Footprint Network (2021), os cidadãos do planeta Terra estão exigindo quatro vezes mais recursos que nosso planeta pode regenerar, ou seja, existe um déficit de recursos naturais que está refletindo diariamente em diversas catástrofes naturais e prejuízos para saúde e qualidade de vida das pessoas.

Em função do uso dos recursos naturais em grandes proporções, muito se discute sobre a consciência e o comprometimento com o desenvolvimento sustentável (TÚLIO; TÚLIO, 2018), aquele no qual seria possível suprir as necessidades da população atual e ao mesmo tempo garantir com que as futuras gerações possam suprir suas necessidades sobre os recursos do planeta (BRUNDTLAND, 1991).

Com esse objetivo de alcançar o Desenvolvimento Sustentável, a Economia Circular surgiu como sendo uma nova maneira de se produzir e consumir em ciclos fechados (PRIETO SANDOVAL; JACA; ORMAZABAL 2018). Ela tem como objetivo redefinir a noção de crescimento, trazendo benefícios para toda a sociedade, fazendo com que produtos atinjam altos níveis de desempenho, porém planejando-os para a remanufatura e eliminando resíduos e poluição gerados em sua produção (ELLEN MACARTHUR FOUNDATION, 2021). Diante disso, nos últimos anos os padrões de produção têm se modificado (SMITH; OFFODILE, 2014), devido à necessidade da adoção de um cenário voltado para a sustentabilidade (LLORET, 2016). Na indústria da construção civil, segundo Túlio e Túlio (2018), os recursos naturais renováveis e não-renováveis são utilizados em grande quantidade, tais como água, madeira, pedras, areia, o que acarreta diversos impactos ambientais.

De acordo com Plataform for Accelerating the Circular Economy - PACE (2019), o setor da construção civil é responsável por $50 \%$ do uso de materiais globalmente (42,4 bilhões de toneladas) e segundo Goldemberg et al. (2011), a propagação de tecnologias ecoeficientes reduz significativamente os impactos ambientais causado pela construção civil, sendo assim teríamos que reinventar padrões de construção e buscar inovações para esse mercado.

Diante dessa problemática, surge a necessidade do desenvolvimento e uso de produtos ecológicos e sustentáveis na indústria da construção civil. Assim sendo, com este trabalho 
buscou-se identificar os principais desafios para o desenvolvimento e utilização de produtos sustentáveis na construção civil.

\title{
2. REVISÃO TEÓRICA
}

\subsection{Desenvolvimento sustentável e sustentabilidade}

Segundo Van Bellen (2006), a sociedade começou a refletir sobre os padrões de vida exagerados e incompatíveis com a recuperação do meio ambiente, voltando-se a um apelo de conscientização sobre os problemas ambientais na década de 1970.

Ainda segundo Van Bellen (2006), na mesma década, surge o termo "ecodesenvolvimento", após a primeira Conferência da Organização das Nações Unidas (ONU) sobre o Meio Ambiente, realizada em Estocolmo. O termo foi proposto inicialmente por Mauricie Strong, secretário da Conferência de Estocolmo, em 1973 e, em seguida, ampliado pelo economista Ignacy Sachs (VAN BELLEN, 2006).

O Desenvolvimento Sustentável pode ser conceituado como:

\begin{abstract}
Um processo de transformação no qual a exploração dos recursos, a direção dos investimentos, a orientação do desenvolvimento tecnológico e a mudança institucional se harmonizam e reforça o potencial presente e futuro, a fim de atender às necessidades e aspirações futuras (...) é aquele que atende às necessidades do presente sem comprometer a possibilidade de as gerações futuras atenderem as suas próprias necessidades (WORLD COMMISSION ON ENVIRONMENT AND DEVELOPMENT - WCED, 1987, p. 46).
\end{abstract}

Stoffel e Colognese (2015) defendem que o conceito de Desenvolvimento Sustentável se tornou uma referência para a sociedade contemporânea, sendo necessárias as preocupações com as questões que os diversos segmentos da sociedade estabelecem em relação ao crescimento econômico e à preservação dos recursos naturais. Com isso, o desenvolvimento sustentável deve ser além de um discurso para as futuras gerações, mas uma tarefa para ser executada no momento atual (ARRUDA; QUELHAS, 2010).

Um termo que guarda forte relação com o desenvolvimento sustentável é a sustentabilidade (CARVALHO, 2019). São muitas as definições que foram elaboradas para sustentabilidade ao longo das últimas décadas, mas existe um alinhamento conceitual no sentido de trazer a necessidade de produção com impactos ambientais negativos reduzidos devido, em parte, à redução na exploração dos recursos naturais e na geração de resíduos (CIOFI, 2010). A disseminação do conceito de sustentabilidade tem contribuído para gerar cobranças mais intensas da sociedade sobre o setor produtivo (CIOFI, 2010). 


\subsection{Economia circular}

Para Barboza et al. (2019), a economia circular pode ser vista como uma opção à abordagem convencional de economia linear na qual os recursos são usados para uma única finalidade dentro da cadeia produtiva, sendo descartados ao longo do processo industrial ou ao final do ciclo de vida do produto pelo consumidor. A Economia Linear, tem como características a produção a partir de matérias-primas virgens, com o consumo e o posterior descarte dos produtos, como resíduos, quando obsoletos (ELLEN MACARTHUR FOUNDATION, 2012). O sistema de produção circular é um conceito no qual a palavra "desperdício" passa a ser relativizada, já que em sua proposta o que hoje se descarta, e na maioria das vezes é desperdiçado na forma de resíduo, passa a ser reinserido em um novo ciclo de produção, recebendo agregação de valor (BARBOZA et al., 2019). O propósito da Economia Circular é criar projetos de berço a berço, que consistem em conceber produtos e sistemas industriais que sejam capazes de manter a qualidade e produtividade dos materiais em ciclos de vidas seguintes (BERNDTSSON, 2015). Este conceito baseia-se em três princípios fundamentais: A eliminação do conceito de resíduo, o uso de energias com fontes renováveis (principalmente a energia solar) e a gestão do uso da água que promova ecossistemas saudáveis e respeite os impactos locais (ELLEN MACARTHUR FOUNDATION, 2013). Assim a Ellen Macarthur Foundation (2017) propõe um sistema de Economia Circular, no qual haja regeneração no ciclo com a gestão do fluxo de bens renováveis para que estes possam se recuperar em tempo hábil para seguirem sendo utilizados de maneira sustentável, ou seja, atrelada à gestão do ciclo para garantir o prolongamento de estoques de maneira a minimizar perdas sistêmicas e externalidades negativas. Na concepção do modelo apresentado na figura 1, os produtos, segundo a Ellen Macarthur Foundation (2013), podem ser divididos em dois grupos: os biológicos, projetados para serem inseridos de novo na natureza e os técnicos, que necessitam de investimentos em inovação para serem desmontados e reinseridos nos ciclos. Para não gerar resíduos durante e ao final da produção, materiais que não podem ser reutilizados são descartados antes de começar o processo, dando lugar a materiais biológicos não tóxicos que podem ser reinseridos na natureza sem causar danos e ainda gerar benefícios ao meio ambiente (ELLEN MACARTHUR FOUNDATION, 2012). 


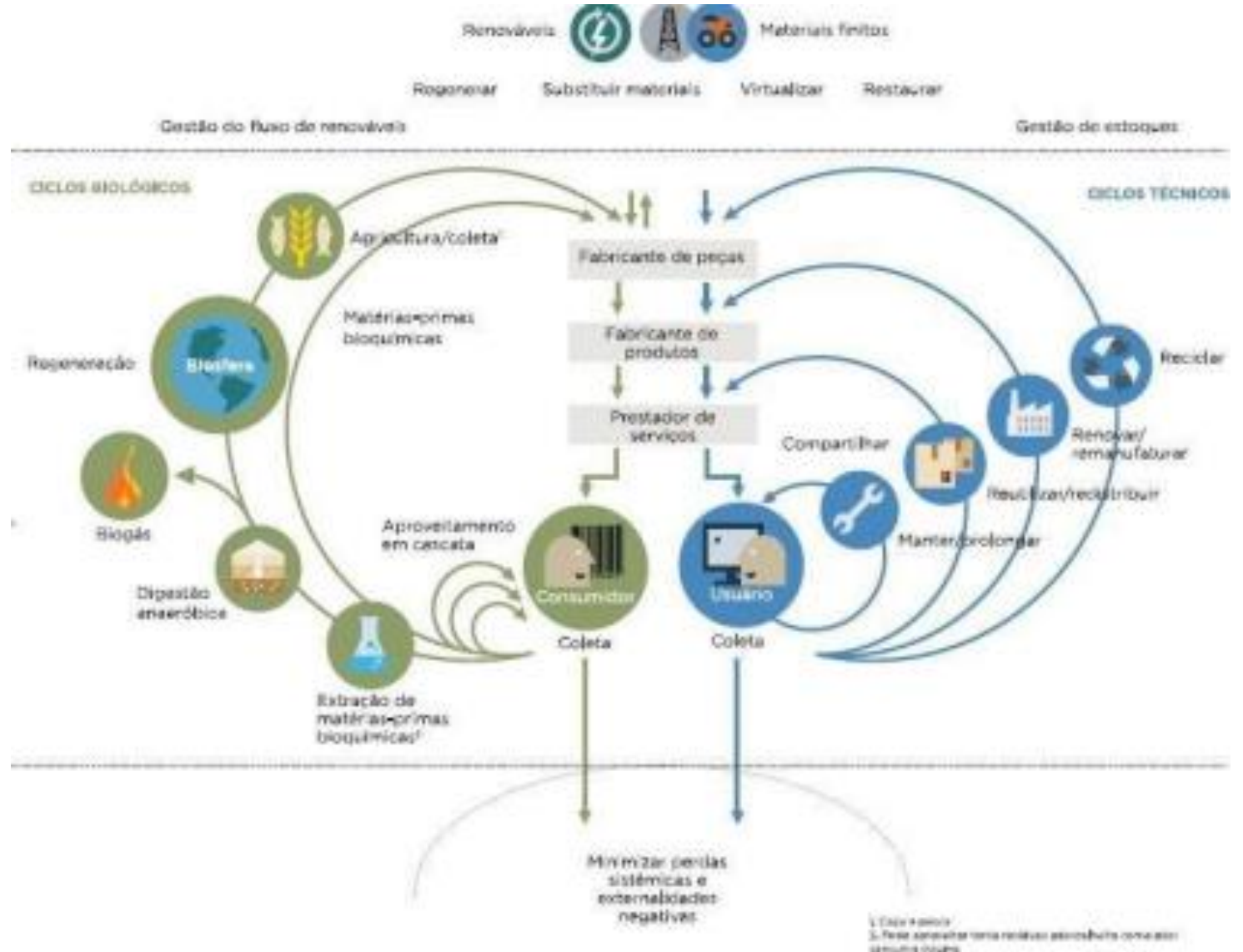

FIGURA 1 - Modelo de economia circular. Fonte: Ellen Macarthur Foundation (2013).

\subsection{Inovação para sustentabilidade na construção civil}

Os requisitos de sustentabilidade têm aplicado uma série de aperfeiçoamentos nas metodologias da construção civil, segundo Goldemberg et al. (2011). Borges e Marco (2020) afirmam que repensar os métodos construtivos em todo o seu processo, garante uma nova visão dos problemas de resíduos da construção civil buscando novas inovações, trazendo assim ganhos efetivos para todo o setor, tais como melhoria do controle de custos, e qualidade dos processos. As práticas sustentáveis durante todo o processo construtivo fazem com que se tenha a redução de resíduos gerados, como os materiais, energia, água e outros recursos naturais, com esse planejamento de sustentabilidade desde o projeto ao uso da edificação resultando em grandes benefícios para as partes envolvidas (BORGES; MARCO, 2020).

\section{METODOLOGIA}

A metodologia utilizada neste estudo para identificar os desafios do desenvolvimento de produtos sustentáveis na construção civil, teve uma base descritiva (GIL, 2017). Para Gil (2017), a pesquisa descritiva tem como principal objetivo descrever as características de 
determinada população ou fenômeno, podendo também estabelecer relações entre variáveis, sendo incluídas nesse grupo as pesquisas que têm por objetivo levantar as opiniões, atitudes e crenças de uma população, visando descobrir a existência de associações entre variáveis. Quanto à abordagem, trata-se de uma pesquisa com dados qualitativos, pois lidou-se diretamente com os sujeitos que tiveram experiência com o problema pesquisado. Segundo Creswell (2010), a abordagem qualitativa é aquela em que o pesquisador coleta dados emergentes abertos, utilizando-os para desenvolver o tema de estudo. Os principais procedimentos qualitativos implicam primeiramente em classificar coisas, pessoas, eventos e as propriedades que os caracterizam, buscando identificar e descrever padrões e temas a partir da perspectiva dos participantes (CRESWELL, 2010).

As etapas seguidas nesta pesquisa podem ser visualizadas na Figura 2.

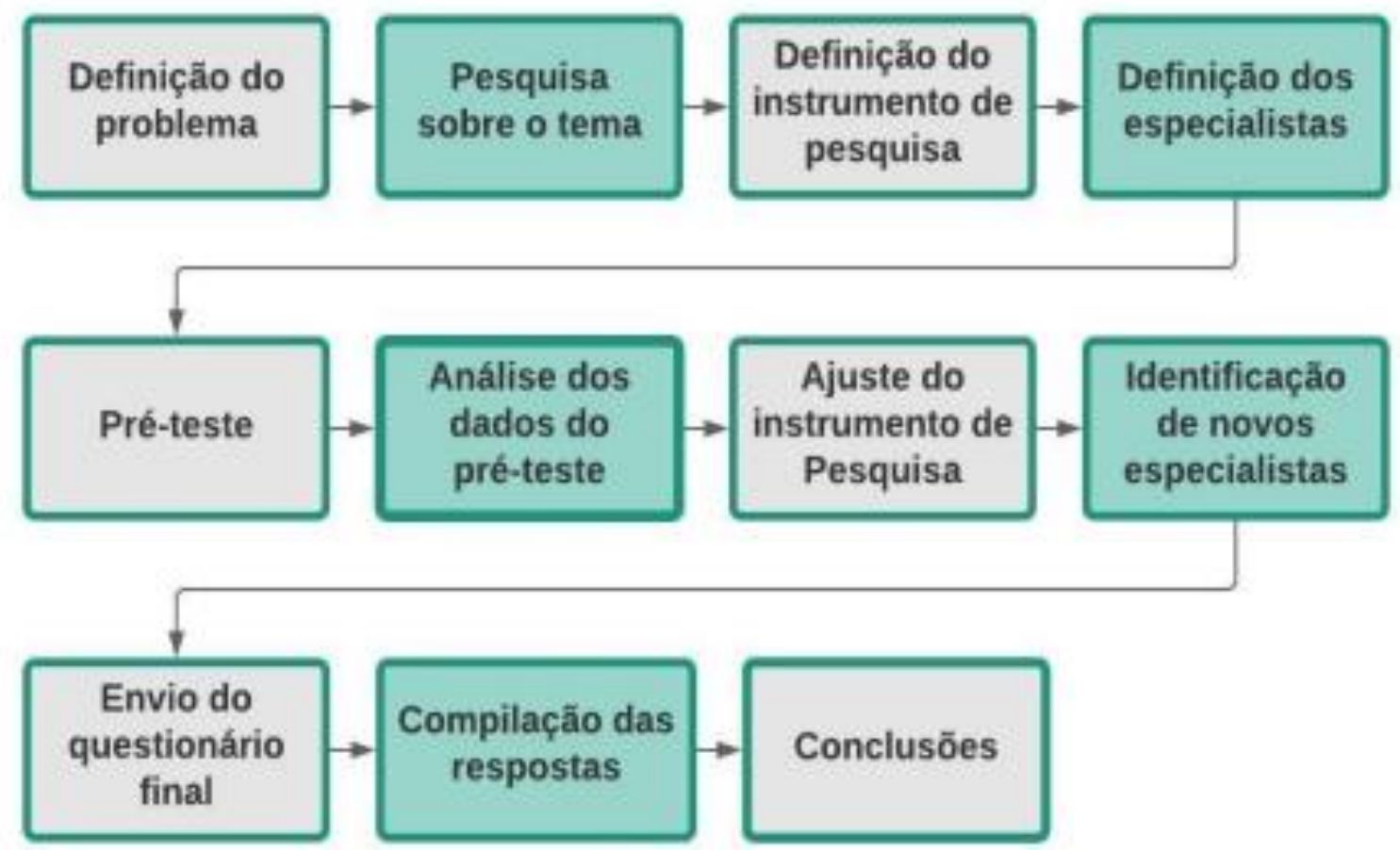

FIGURA 2 - Etapas da pesquisa.

Inicialmente foi identificado o problema através da observação a respeito da sustentabilidade na construção civil. Em seguida, foi feita uma revisão bibliográfica sobre os temas que permeiam a pesquisa, tais como o desenvolvimento sustentável, a economia circular e a inovação e sustentabilidade na construção civil. Após essa revisão, foi elaborado um questionário eletrônico utilizando o Google Forms, para levantar os desafios do desenvolvimento de produtos sustentáveis na para a construção civil. 
Os primeiros cinco especialistas selecionados foram professores e profissionais das áreas de construção civil e desenvolvimento de produtos. Tendo em vista a situação atual de pandemia e distanciamento social, o questionário virtual foi o método viável para a coleta de dados. Antes de realizar uma aplicação mais ampla, foi executado um pré-teste coletando os dados respondidos pelos professores e profissionais especialistas como engenheiros de produção, engenheiros civis e de materiais que estudam sobre temas como economia circular, desenvolvimento e análise de produtos na indústria de construção civil. Essa aplicação foi feita no mês de agosto de 2020, com os resultados analisados na sequência.

Em seguida, foram feitas melhorias ao questionário antes de enviá-lo a profissionais especialistas que atuam diretamente na construção civil com o uso de produtos sustentáveis em seus projetos e bioconstruções, dentre eles arquitetos e engenheiros civis de todo o país. O questionário foi aplicado entre os meses de abril e maio de 2021, alcançando mais 14 participantes, totalizando 19 respondentes entre pré-teste e teste.

Dessa forma, o fato da amplitude de cargos dos entrevistados foi fundamental para a pesquisa proposta, pois possibilitou a identificação sob diferentes óticas do problema proposto. Para tanto, a definição da amostra de pesquisa adotou o critério de conveniência, uma vez que os pesquisadores, além de docentes na área, contam também com profissionais experientes no âmbito das construções sustentáveis. Esses dois contextos foram considerados como focos e determinantes para a aplicação do questionário.

A compilação das respostas foi feita por meio da análise das respostas dos 19 participantes. $\mathrm{Na}$ tabela 1 a seguir são apresentas as perguntas que constam no questionário enviado aos especialistas.

TABELA 1 - Questões que compuseram o questionário aplicado com os especialistas

\section{ID Questão}

Q1 Seria viável o processo de desenvolvimento de produtos sustentáveis a partir do conceito de economia circular para a construção civil? Justifique sua resposta.

Q2 Na sua opinião, quais as principais dificuldades da implementação de produtos sustentáveis na construção civil?

Q3 Quais estudos feitos por meio de testes e softwares sobre características físicas dos produtos sustentáveis poderiam incentivar o uso desses produtos pelos consumidores?

Q4 Quais as tendências futuras para o desenvolvimento de produtos para a área da construção civil? Seria possível que essas tendências de construções ecológicas se tornem prioritárias com o passar dos anos? 


\section{RESULTADOS E DISCUSSÃO}

As respostas foram analisadas individualmente e foi possível identificar quais eram os temas mais citados pelos especialistas ao responder cada questão. Uma síntese das 19 respostas obtidas em cada questão é apresentada na sequência.

$Q_{1}$ : Seria viável o processo de desenvolvimento de produtos sustentáveis a partir do conceito de economia circular para a construção civil? Justifique sua resposta.

Percebeu-se pelas respostas, em sua grande maioria foi que o referido processo de desenvolvimento de produtos é viável, tendo-se como ponto de destaque a necessidade de se identificar os principais gargalos ambientais para se trabalhar de forma consciente, seguindo com isso o conceito de economia circular. Foram mencionadas estratégias como redução no uso de materiais, reuso e reciclagem (3R's). Apontou-se a simbiose industrial e o desenvolvimento de novos modelos de negócios como alternativas importantes.

Alguns especialistas destacaram a necessidade de um olhar mais próximo para a dimensão social da sustentabilidade social, com a escolha e capacitação da mão de obra local, melhores condições de trabalho e a busca por fornecedores que atendam aos requisitos sustentáveis. Foi apontado também como importante a reeducação dos profissionais da área, trazendo conhecimentos sobre o conceito de economia circular e sustentabilidade. Os estudos de viabilidade técnica, econômica e ambiental dos processos de desenvolvimento dos produtos foram apontados como necessários para todos os desenvolvimentos.

Eles acreditam que com a disseminação desses conceitos e com a reeducação dos profissionais da indústria da construção civil, a demanda por produtos mais sustentáveis seria ampliada. Isso conduziria a economias de escala, com consequentes reduções de custos e de impactos ambientais negativos.

$Q_{2}$ : Na sua opinião, quais as principais dificuldades da implementação de produtos sustentáveis na construção civil? 
Nas respostas a este questionamento, notou-se que a principal dificuldade apontada pelos especialistas foi a falta de aceitação dos produtos sustentáveis pelos profissionais do mercado da construção civil e pelos consumidores. Foi apontado que esta relação existe por conta da mentalidade predominante na indústria, baseada em produtos tradicionais. Outras causas que justificam a baixa aceitação são: a falta de conhecimento por parte de profissionais e clientes e a existência de poucas pesquisas sobre os produtos sustentáveis. Outro fator fundamental diz respeito ao fato de esses produtos não terem custos competitivos como os convencionais.

De acordo com Ferreira e Flach (2020) existem dois tipos de consumidores, os que acreditam que os problemas ambientais são severos e compram com responsabilidade ambiental e os que só compram produtos sustentáveis se tiverem o mesmo preço do produto comum, sendo esse um desafio para que novos produtos sustentáveis sejam desenvolvidos e venham a ser competitivos no mercado.

Foi apontada ainda a dificuldade de implementação de novos produtos sustentáveis devido ao oligopólio de empresas do setor. Essas empresas são capazes de dominar e influenciar o mercado em que atuam, impedindo a entrada de novas empresas e prejudicando o consumidor com a falta de concorrência e acesso a produtos inovadores (ALMEIDA, 2019). E, por fim, destacam a falta de incentivos governamentais para o desenvolvimento de tais produtos.

Q3: Quais estudos feitos por meio de testes e softwares sobre características físicas dos produtos sustentáveis poderiam incentivar o uso desses produtos pelos consumidores?

Na maioria das respostas não se elencou um estudo específico sobre características físicas, pois eles variam muito de acordo com cada produto. Os especialistas apontaram análises do produto sustentável durante todo o seu uso, ou seja, análise do ciclo de vida do produto e outros estudos como durabilidade, resistência, índice de regulamentação térmica e higrometria. Algumas respostas sugeriram protótipos de produtos, que incentivariam o uso de tais materiais, mostrando suas características na prática aos consumidores.

Já outros especialistas acreditam que esses estudos por meio de testes e softwares sobre características físicas dos produtos sustentáveis não influenciarão o uso desses produtos pelos consumidores, indicando que o fator preço é preponderante na escolha do consumidor. 
Q4: Quais as tendências futuras para o desenvolvimento de produtos para a área da construção civil? Seria possível que essas tendências de construções ecológicas se tornem prioritárias com o passar dos anos?

A conclusão sobre as respostas foi que em grande maioria acredita-se que será possível que as construções ecológicas se tornem prioritárias, mas com ressalvas. Elas só serão possíveis se esses produtos tiverem passado por estudos preliminares em relação a custos e características físicas de desempenho, sendo necessário também o desenvolvimento de normas para tais produtos, sem esquecer a importância da educação ambiental para todos desde as séries iniciais nas escolas.

Citaram o bambu como principal tendência de matéria-prima e a manufatura aditiva como principal processo de fabricação nos próximos anos. Mencionou-se também sobre a manutenção de territórios sustentáveis que devem ser utilizados com maior frequência e o necessário estudo sobre o uso de recursos locais.

Embora com o ecológico seja uma tendência, foi colocado como se ele não fosse democratizado, afinal apenas pessoas com alto poder econômico possuem acesso a esses produtos, sendo assim, seriam necessários incentivos econômicos governamentais para o setor da construção civil, que tornariam essas construções sustentáveis minimamente competitivas.

\section{CONCLUSÃO}

Com a limitação de recursos naturais e a necessidade de utilização dos produtos sustentáveis, esses produtos tornam-se indispensáveis para a indústria da construção civil, devido à necessidade da adoção de um cenário voltado para a sustentabilidade, pois o planeta está em déficit de recursos naturais.

Esse trabalho objetivou encontrar os maiores empecilhos para a utilização de produtos sustentáveis na construção civil e teve como resultado que o principal desafio é o preço dos produtos, pois a falta de educação ambiental por parte dos consumidores e profissionais faz com que não se tenha sua utilização em grande escala, fazendo com que se tenha um alto custo de produção que é repassado diretamente ao consumidor. 
Para se ter uma nova realidade, é necessária uma reeducação de todo o setor, através de estudos sobre características físicas de produtos sustentáveis, seu ciclo de vida, análise de seus custos e elaboração de normas técnicas específicas para eles.

Com esses estudos e testes de desempenho, o setor deixaria de ter o preconceito com esses itens e aumentaria o seu consumo, consequentemente reduzindo o custo de produção e trazendo um produto totalmente competitivo em relação a preço e desempenho, mas com um diferencial, pois seguiria o conceito de economia circular e sustentabilidade.

As limitações principais desta pesquisa são relacionadas à quantidade de respostas obtidas com o questionário e que a maior parte das respostas são oriundas de um mesmo grupo de profissionais da área, embora estes estejam distribuídos por todo o país e em diversas realidades de implementação de produtos sustentáveis na construção civil.

\section{AGRADECIMENTOS}

Deixamos nossos sinceros agradecimentos à Rede Terra Brasil e aos outros especialistas envolvidos pela disponibilidade e colaboração com a nossa pesquisa.

\section{REFERÊNCIAS}

ALMEIDA, S. S. Estruturas de mercado: como as empresas oligopólios formam seus preços? Revista Científica Multidisciplinar Núcleo do Conhecimento, São Paulo, v. 9, n. 3, p. 42-68, 2019.

ARRUDA, L.; QUELHAS, O. L. G. Sustentabilidade: um longo processo histórico de reavaliação crítica da relação existente entre a sociedade e o meio ambiente. Boletim Técnico do Senac, Rio de Janeiro, v. 36, n. 3, p. 53-63, 2010.

BARBOZA, D. V. et al. Aplicação da economia circular na construção civil. Research, Society and Development, v. 8, n. 7, p. e9871102, 16 maio 2019. DOI: http://doi.org/10.33448/rsd-v8i7.1102.

BERNDTSSON, M. Circular economy and sustainable development. 2015. Dissertação (Mestrado em desenvolvimento sustentável) - Department of Earth Sciences, Uppsala University, Uppsala.

BORGES, M. V. J.; MARCO, G. Sustentabilidade na construção civil: técnicas construtivas. Revista Científica Semana Acadêmica. Fortaleza, v. 8, n. 201, 2020.

BRUNDTLAND, G. H. Nosso futuro comum: comissão mundial sobre meio ambiente e desenvolvimento. 2. ed. Rio de Janeiro: Fundação Getúlio Vargas, 1991.

CARVALHO, G. O. Sustentabilidade e desenvolvimento sustentável: uma visão contemporânea. Revista Gestão e Sustentabilidade Ambiental, v. 8, p. 779-792, jan/mar. 2019.

CIOFI, J. L. Uma investigação do nível de sustentabilidade das companhias de papel e celulose e a influência das informações financeiras sobre a qualidade da divulgação socioambiental. 2010. Dissertação (Mestrado em Controladoria e Contabilidade) - Faculdade de Economia, Administração e Contabilidade de Ribeirão Preto, Universidade de São Paulo, Ribeirão Preto, 2010.

CRESWELL, J. W. W. Projeto de pesquisa: métodos qualitativo, quantitativo e misto. 2. ed. Porto Alegre: Bookman, 2010.

ELLEN MACARTHUR FOUNDATION. Towards the circular economy 1: economic and business rationale for an accelerated transition. Cowes, Isle of Wight: Ellen MacArthur Foundation, 2012. 
ELLEN MACARTHUR FOUNDATION. Growth within: a circular economy vision for a competitive Europe, 2013. Disponível em: https://www.ellenmacarthurfoundation.org/assets/downloads/publications/EllenMac ArthurFoundation_Growth-Within_July15.pdf. Acesso em: 30 ago. 2020.

ELLEN MACARTHUR FOUNDATION. Economia circular, 2017. Disponível em: https://www.ellenmacarthurfoundation.org/pt/economia-circular/conceito. Acesso em: 02 mar. 2021.

ELLEN MACARTHUR FOUNDATION. A Recuperação da Covid-19 requer uma economia circular resiliente, 2021. Disponível em: https://medium.com/circulatenews/the-covid-19-recovery-requires-a-resilient circular-economy-e385a3690037. Acesso em: 02 mar. 2021.

FERREIRA, T. L.; FLACH, R. O. Preocupação ambiental, intenção de compra e compra declarada de produtos sustentáveis: um estudo empírico. In: SIMPÓSIO INTERNACIONAL DE GESTÃO DE PROJETOS, INOVAÇÃO E SUSTENTABILIDADE, 8., 2020, São Paulo. Anais, 2020.

GIL, A. C. Como elaborar projetos de pesquisa. 6. ed. São Paulo: Atlas, 2017.

GLOBAL FOOTPRINT NETWORK. Footprint Network, 2021. Disponível em: https://data.footprintnetwork.org/?_ga=2.256181131.1068005558.1617241114-1944573688.1617241114\#/ Acesso em: 02 mar. 2021.

GOLDEMBERG, J. O desafio da sustentabilidade na construção civil. São Paulo: Blucher, 2011.

LLORET, A. Modeling corporate sustainability strategy. Journal of Business Research, v. 69, n.2, p. 418-425, 2016.

PLATAFORM FOR ACCELERATING THE CIRCULAR ECONOMY. The circularity gap report 2019: closing the circularity gap in a 9\% world, 2019. Disponível em: <https://bfc732f7-80e9-4ba1-b4297f76cf51627b.filesusr.com/ugd/ad6e59_ba1e4d16c64f44fa94fbd8708eae8e34.pdf>. Acesso em: 02 mar. 2021.

PRIETO-SANDOVAL, V.; JACA, C.; ORMAZABAL, M. Towards a consensus on the circular economy. Journal of Cleaner Production, v. 179, p. 605-615, 2018.

SMITH, A. D.; OFFODILE, O. F. Green and sustainability corporate initiatives: a case study of goods and services design. International Journal of Logistics Systems and Management, v. 6, n. 3, p. 273, 2014.

STOFFEL, J. A. COLOGNESE, S. A. O Desenvolvimento sustentável sob a ótica da sustentabilidade multidimensional. Rev. FAE, Curitiba, v. 18, n. 2, p. 18 - 37, jul./dez. 2015.

TÚliO, F.; TÚliO, L. Gestão de projetos sustentáveis. Ponta Grossa: Atena Editora, 2018. Disponível em: https://www.atenaeditora.com.br/wp-content/uploads/2018/10/E-book-Gest\%C3\%A3o-de-Projetos

Sustent\%C3\%A1veis.pdf. Acesso em: 02 mar. 2021.

VAN BELLEN, H. M. Indicadores de sustentabilidade: uma análise comparativa. 2. ed. Rio de Janeiro: FGV, 2006.

WORLD COMMISSION ON ENVIRONMENT AND DEVELOPMENT. Our common future. Oxford: Oxford University Press, 1987. Disponível em: https://bfc732f7-80e9-4ba1-b4297f76cf51627b.filesusr.com/ugd/ad6e59_ba1e4d16c64f44fa94fbd8708eae8e34.pdf. Acesso em: 18 mar. 2021. 